\title{
ESTRATEGIA METODOLOGICA PARA LA ENSEÑANZA-APRENDIZAJE DE LA TECNICA DEL GOLPEO DEL BALÓN CON EL PIE EN EL FÚTBOL
}

\author{
Zambrano. T. ${ }^{1}$ Vera. J. ${ }^{2}$ Merchan. $\mathrm{R}^{3}$ Jaimes. $M{ }^{4}$ \\ 1. Zambrano Tony (2016). Licenciado en Educación Física. Aspirante a magister en ciencias de la actividad física y \\ deporte Universidad de Pamplona tony2@hotmail.com \\ 2. Vera José Luis (2016). Doctor en ciencias de la cultura física, profesor universidad del valle, Grupo de \\ Investigación Grinder vera.jose@correounivalle.edu.com.co \\ 3. Merchan Rony (2016). Magister en Actividad Física, Docente Investigador Fundación Universitaria Juan de \\ Castellanos. rdmerchan@jdc.edu.co \\ 4. Marco Fredy Jaimes laguado ${ }^{3}$ Doctor en Nuevas Perspectivas de Investigación en Ciencias de la Actividad \\ Física y del Deporte, Profesor Universidad de Pamplona, Grupo de Investigación actividad Física y Deportes. \\ mafrejala@ugr.es.
}

\section{RESUMEN}

El presente trabajo tuvo como propósito diseñar una estrategia metodológica basada en la enseñanza-aprendizaje fusionando los métodos analítico y global los cuales nos sirvieron para planificar el macrociclo de trabajo con sus respectivos, mesociclos, microciclos y unidades de trabajo diario. Este trabajo de investigación es de perfil casiexperimental cuya población son 450 niños que pertenecen a la institución educativa COLEGIO INTEGRADO PETROLEA; para la selección de la muestra se realizó mediante la modalidad no probabilística seleccionando así niños los cuales cumplían con las características necesarias para llevar a cabo esta investigación. El principal criterio para la selección de los niños fue tener más de 7 años de experiencia laborando en distintas escuelas de formación deportiva en futbol. Luego de terminada la intervención de 5 meses a través de los mesociclos con sus respectivos propósitos, se concluyó que, la aplicación de esta propuesta metodológica obtiene resultados significativos en cuanto al mejoramiento de la técnica del golpeo del balón con las principales superficies de contacto de las dos piernas. Se recomienda a los encargados de las escuelas de formación deportiva hacer mucho más énfasis en la técnica ya que sin este aspecto no se podría llevar a cabo un trabajo táctico acorde a las necesidades de un partido de futbol.

Palabras clave: futbol, técnica, golpeo del balón con el pie, enseñanza-aprendizaje del futbol, niños.

\begin{abstract}
The present work was aimed to design a methodological strategy based on teaching and learning merging analytical and comprehensive methods which helped us to plan the macrocycle work with their respective mesocycles, Microcycles and daily work units. This research is quasi-experimental profile whose population is 450 children belonging to the school INTEGRATED SCHOOL PETROLEA; for the selection of the sample was performed by the non-probabilistic mode so selecting children who met the necessary features to conduct this research. The main criterion for selection of children was having more than 7 years experience working in different schools of sports training in football. After completion of the intervention of five months through mesocycles with their respective purposes, it was concluded that the application of this methodological proposal obtained significant results in terms of improving stroke technique of the ball with the main contact surfaces of the two legs. those in charge of sports training schools are recommended much more emphasis on technique because without this aspect could not conduct a tactical work according to the needs of a football match.
\end{abstract}

Keywords: Soccer, technique, kick, learning education the soccer, kids.

Convocatoria III. Trimestre. Recibido el 15 de junio de 2017; Aceptado el 15 de julio de 2017. 


\section{INTRODUCCIÓN}

El futbol con el correr de los años se ha convertido en el deporte más popular del mundo. $Y$ eso se ve reflejado en muchos aspectos como por ejemplo en los estratos sociales; la mayoría de las personas que aparentemente están bien de salud practican el futbol deporte en el cual uno puede ver en el campo de juego personas de diferentes clases sociales y razas. Lo cual lleva a este deporte a adquirir una vital importancia si se le da el rumbo adecuado para la integración de toda la humanidad.

Otro aspecto en el cual se puede evidenciar la popularidad de este deporte es cuando inicia el mundial de futbol; prácticamente el planeta se paraliza debido a la gran expectativa que genera el campeonato más importante a nivel de selecciones.

En Colombia, y más exactamente en Norte de Santander, el futbol es la disciplina deportiva más practicada lo cual para nadie es un secreto, pero también hay que decir que este deporte en muchos casos se practica de forma empírica lo cual lleva a los jugadores a tener problemas con la técnica, la táctica y la condición física a la hora de enfrentar un partido de futbol.

En los países en vía de desarrollo como el nuestro, los jugadores cuando están en la etapa de la pubertad, en muchos casos ven en el futbol una alternativa para ayudar a sacar adelante a su familia; y de hecho en Colombia hay infinidad de casos en los cuales eso ha ocurrido; uno muy mencionado es el de FAUSTINO ASPRILLA tal vez uno de los jugadores más representativos de nuestro país a nivel internacional; $y$ así se pueden encontrar muchos. Lo cual nos lleva a pensar que, si esos potenciales jugadores profesionales se les ayuda en lo que tiene que ver con una adecuada formación técnico táctica y física, nuestro país podría exportar muchos más jugadores de los que están saliendo hoy en día al exterior, en consecuencia, se le mejoraría la situación a muchas familias que son de estratos bajos.

Por otra parte, cabe resaltar que Colombia tanto en divisiones menores como en selección absoluta ha clasificado a varios mundiales y por ejemplo la selección sub 20 logro obtener el campeonato de esperanzas de Toulon.

\section{MARCO METODOLOGICO}

\section{MÉTODO}

Es del orden inductivo-deductivo ya que vamos de lo general que es el futbol a lo particular que son los gestos técnicos del golpeo del balón con el pie.

INSTRUMENTOS: se realizó una depuración con la ayuda de expertos en futbol y se llegó a la conclusión de que los test más adecuados para evaluar la técnica del golpeo del balón son los siguientes: consisten en dos test específicos para evaluar el golpeo del balón con borde interno y empeine de la pierna izquierda y derecha.

Uno es un test de tiro a portería, del profesor ROBERTO MONTES GARCIA.

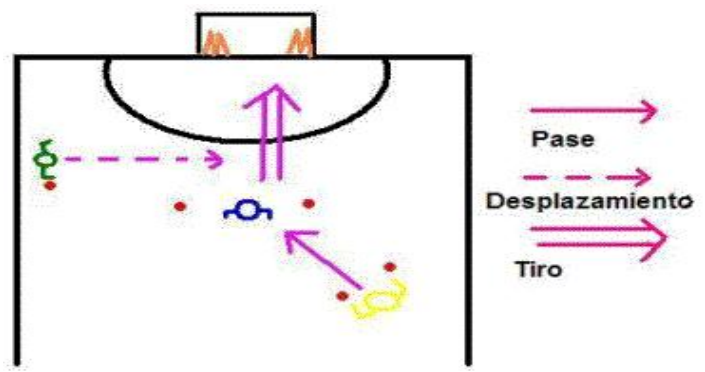

\section{DESCRIPCION:}

El jugador azul, será en este caso el que va a ser evaluado, partirá de una posición de espaldas a la portería, aproximadamente a tres metros del área. Un compañero, (jugador amarillo) se situará con balón a unos 7 metros más retrasado que el jugador azul y en banda derecha. El jugador verde, se situará en la línea de banda izquierda a la altura del jugador verde. Por último, en la portería se situarán dos conos junto a cada palo y un portero entre los conos.

El test comienza cuando el jugador amarillo realiza un pase al pie al jugador azul, el cual debe realizar un tiro a portería desde fuera del área antes de que el jugador verde (que empieza a correr en el momento en que el jugador amarillo realiza el pase), le alcance e intercepte el tiro.

Cada jugador realizará 5 tiros recibiendo el 
pase desde la derecha como se explica en el dibujo anterior, y 5 tiros recibiendo el pase por la izquierda, saliendo el defensor desde el lado contrario.

El jugador azul (el cuál es evaluado), debe de conseguir realizar el tiro de forma rápida, de manera que logre golpear al balón sin que el jugador le moleste, por lo que esta será la primera valoración que hagamos del jugador (velocidad de tiro). Para evaluar los otros dos parámetros (precisión y potencia), hemos colocado un objetivo en el interior de la portería (4 conos, 2 junto a cada poste), los cuáles los jugadores deben intentar derribar, de tal forma que se combine en el golpeo a portería tanto la precisión (tocar el cono), como la potencia (derribar el cono).

Sistema de puntuación

El jugador no logra: 1

El defensor intercepta: 2

El tiro se va por fuera: 3

El tiro lo detiene el portero: 4

El tiro entra a gol: 5

El tiro entra a gol y derriba el: 6

De los 5 tiros realizados recibiendo el pase desde la derecha, se eliminará tanto la mejor puntuación como la peor de cada jugador, haciendo la media de las otras 3 puntuaciones, la cual será la puntuación final. Lo mismo se realizará con las puntuaciones obtenidas desde el lado contrario, por lo que tras realizar el test cada jugador obtendrá dos puntuaciones, una por cada lado.

Para evaluar el golpeo con la parte interna del pie, se encuentra el test técnico para futbol de la UNIVERSIDAD DEL VALLE, el cual sirve para evaluar la técnica del pase a corta y larga distancia.

\section{$\stackrel{20 \mathrm{~mm}}{1}$}

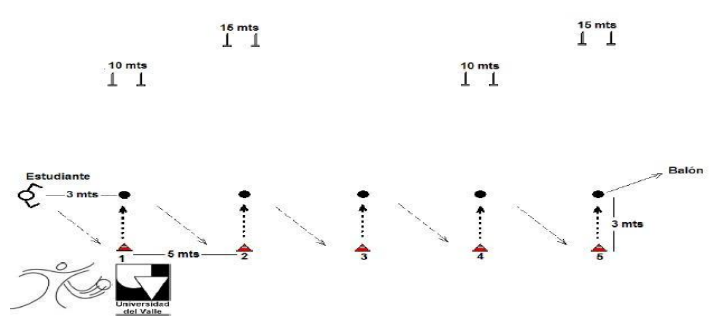

ObjetivoMedir la habilidad del estudiante para realizar pases en corta y larga distancia lo más rápido posible.

MaterialesCinco balones de fútbol, 10 platos, 10 estacas y un cronometro.

Desarrollo El estudiante se sitúa en el lugar de partida, que se encuentra a 3 modelo primer balón, a la orden del evaluador (Monitor), el estudiante deberá correr hacia el primer plato y después correr hacia el primer balón, que están separados $3 \mathrm{~m}$. y realizar el pase, después correr hacia el 2do plato y correr hacia el 2dobalón y realizar el pase, esto se deberá realizar hasta el último balón. El tiempo se detendrá cuando el estudiante lance 0 haga el último pase. El tiempo que dura la prueba por estudiante es de $15 \mathrm{~s}$. La distancia entre estaca y estaca será de $1 \mathrm{~m}$ (esta formará una pequeña portería). Cada anotación tendrá un valor.

Calificación del test

Cada portería tendrá un valor en porcentaje de acuerdo a la distancia que se encuentra con respecto al balón, es decir la primera portería que está ubicada a $10 \mathrm{~m}$. De distancia con respecto al primer balón tendrá un valor del $15 \%$, la 2 da del $20 \%$, la 3 ra del $30 \%$, la 4 ta del $15 \%$, y la 5 ta del $20 \%$.

El valor que tendrán los porcentajes será de:

\begin{tabular}{|l|l|l|}
\hline$\%$ & EFECTIVO & NO EFECTIVO \\
\hline $30 \%$ & 1.5 & 0 \\
\hline $20 \%$ & 1 & 0 \\
\hline $20 \%$ & 1 & 0 \\
\hline $15 \%$ & 0.75 & 0 \\
\hline $15 \%$ & 0.75 & 0 \\
\hline Total & 5 & 0 \\
\hline
\end{tabular}




\section{VALORACION CUALITATIVA DEL GOLPEO DEL BALON}

Debido a que la investigación que de realizo es mixta, con la consulta de dos expertos en futbol ( ver anexo 7), se creó una tabla de valoración CUALITATIVA, en la cual se evidenciara en 3 calificativos la ejecución de las distintas acciones motrices que componen el gesto técnico para golpear el balón, estos son:

ADYACENCIA(A): el sujeto ejecuta la acción motriz con bastante dificultad, en consecuencia, el gesto técnico es ineficiente,

EVOLUCION (E): el sujeto ejecuta la acción motriz, con alguna dificultad, el gesto técnico no resulta tan fluido, pero en general es eficiente.

CONFECCION (C): el sujeto ejecuta la acción motriz correctamente, haciendo que el gesto técnico se vea fluido y a su vez eficiente.

Con base a esto se presenta, en concordancia con la edad y la dificultad de la acción motriz, la calificación ideal a la hora de ejecutar el gesto técnico.

\section{GOLPEO DEL BALON CON EMPEINE}

\begin{tabular}{|c|c|c|c|c|c|}
\hline$\overline{\mathrm{ACClO}}$ & DNES & & $\bar{A}$ & $E$ & C \\
\hline $\begin{array}{l}\text { POSIC } \\
\text { TRON }\end{array}$ & $\begin{array}{l}\text { ION } \\
\text { CO }\end{array}$ & & & & \\
\hline CO $\mid R E$ & ELAC & & & & \\
\hline \begin{tabular}{l|l}
$\mathrm{N}$ & 10
\end{tabular} & $\mathrm{~N}$ & & & & \\
\hline $\begin{array}{l}\text { BA } \\
\text { LO } \\
\mathrm{N}\end{array}$ & & & & & \\
\hline $\begin{array}{l}\text { MOVII } \\
0\end{array}$ & UIEN & & & & \\
\hline IMPUL & D & & & & $x$ \\
\hline so & $E$ & & & & \\
\hline PIERN & $\mathrm{A} \mathrm{HA}$ & & & & \\
\hline $\begin{array}{l}\text { POSIC } \\
\text { DE }\end{array}$ & $\overline{I O N}$ & & & & \\
\hline $\begin{array}{l}\text { AP } \\
\text { OY }\end{array}$ & & & & & $X$ \\
\hline & & & & & \\
\hline
\end{tabular}

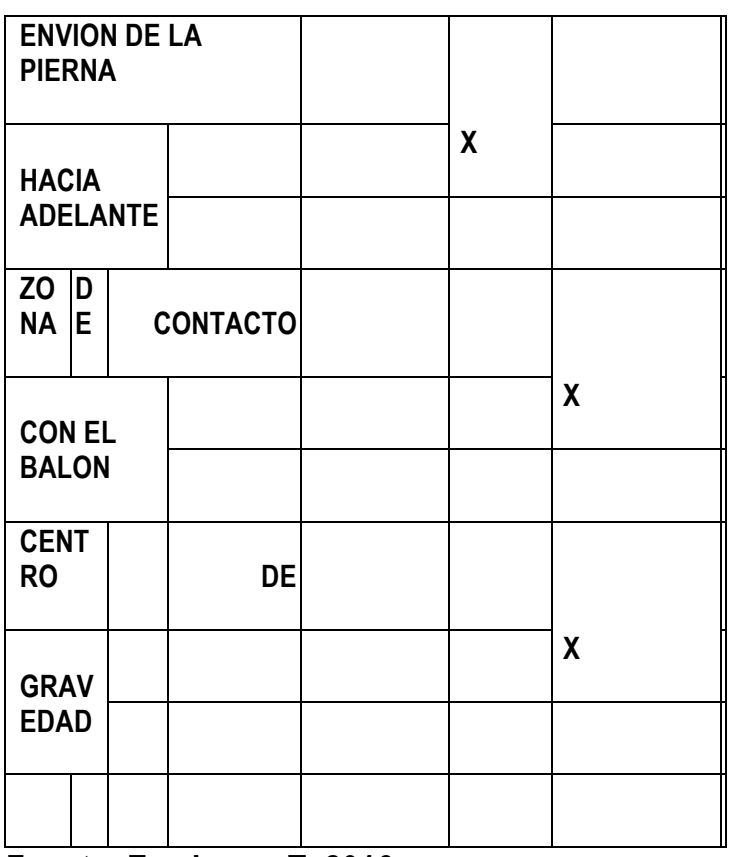

Fuente: Zambrano T. 2016

\section{RESULTADOS}

Para probar la hipótesis de prestest y postest se aplicó la prueba de rangos de signos de Wilcoxon, la cual toma en cuenta tanto el signo como la magnitud de las diferencias, entre cada par de observaciones. La estadística de la prueba de Wilcoxon, calcula rangos negativos, positivos y empates. El nivel de significancia fijado fue del $5 \%$ y los software estadísticos para analizar los datos fueron el SPSS (paquete estadístico para las ciencias sociales) el Statistix 10.0 y el Excel

A continuación, se presentará la tabla correspondiente a los resultados del pretest para el golpeo del balón con la parte interna de las dos piernas.

\begin{tabular}{|c|c|c|c|c|c|c|c|c|}
\hline \multicolumn{9}{|c|}{$\begin{array}{l}\text { GOLPEO DEL BALON CON PARTE } \\
\text { INTERNA PIE DERECHO }\end{array}$} \\
\hline \begin{tabular}{|l|} 
JUG \\
A 1.5
\end{tabular} & & $1 \%$ & $1 \%$ & $\begin{array}{l}0 . \\
5\end{array}$ & ;\% & 0.5 & $\%$ & TOTA \\
\hline$\overline{\mathrm{N}^{\circ}}$ & $<0$ & $y_{0}$ & 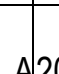 & $0 x$ & 0 & $x$ & 0 & 20 \\
\hline & & & & & & & & \\
\hline \begin{tabular}{|l|l|}
$N^{\circ}$ & $X$ \\
2 & $X$
\end{tabular} & $<0$ & $>0$ & $x 0$ & $A$ & $\begin{array}{l}1 \\
5\end{array}$ & $X$ & 0 & 15 \\
\hline \begin{tabular}{|l|l|}
$N^{0}$ & $X$ \\
3 & $X$
\end{tabular} & $<0$ & $\times 0$ & $A \mid 2$ & $0 x$ & 0 & $A$ & 15 & 35 \\
\hline $\mathrm{N}^{\circ} \mathrm{X}$ & 0 & $A 20$ & $\times 0$ & $\mathrm{~A}$ & 1 & $X$ & 0 & 35 \\
\hline
\end{tabular}




\begin{tabular}{|c|c|c|c|c|c|c|c|c|}
\hline 4 & & & & & 5 & & & \\
\hline $\begin{array}{l}\mathrm{N}^{\circ} \\
5\end{array}$ & 3 & & Y 0 & & & y & 0 & 30 \\
\hline & & & & & & & & \\
\hline $\begin{array}{l}\mathrm{N}^{\circ} \\
6\end{array}$ & $\times 0$ & $>0$ & $\times 0$ & $X$ & 0 & $X$ & 0 & 0 \\
\hline $\begin{array}{l}\mathrm{N}^{\circ} \\
7\end{array}$ & $\times 0$ & $>0$ & $\times 0$ & $x$ & 0 & A & 15 & 15 \\
\hline $\begin{array}{l}\mathrm{N}^{\circ} \\
8\end{array}$ & $x 0$ & $>0$ & $A 20$ & $x$ & 0 & $X$ & 0 & 20 \\
\hline $\begin{array}{l}\mathrm{N}^{\circ} \\
9\end{array}$ & $\times 0$ & $>0$ & $A 20$ & $X$ & 0 & $X$ & 0 & 20 \\
\hline $\begin{array}{l}\mathrm{N}^{\circ} \\
10\end{array}$ & $\mathrm{~A}=3$ & $\times 0$ & $\times 0$ & $x$ & 0 & $X$ & 0 & 30 \\
\hline $\begin{array}{l}\mathrm{N}^{\circ} \\
11\end{array}$ & $\times 0$ & $>0$ & $\times 0$ & $X$ & 0 & $X$ & 0 & 0 \\
\hline $\begin{array}{l}\mathrm{N}^{\circ} \\
12\end{array}$ & $x 0$ & $>0$ & $\times 0$ & $x$ & 0 & A & 15 & 15 \\
\hline $\begin{array}{l}\mathrm{N}^{\circ} \\
13\end{array}$ & $x 0$ & $\times 0$ & $x 0$ & $x$ & 0 & $X$ & 0 & 0 \\
\hline
\end{tabular}

Fuente: Zambrano T. 2016

A continuación, se mostrará la gráfica con los porcentajes de cada jugador

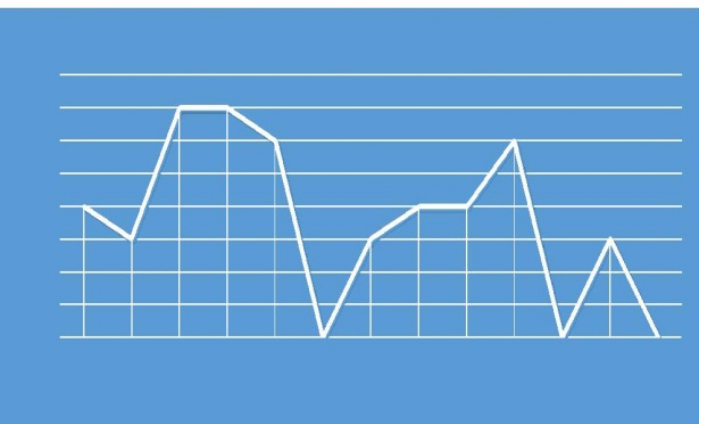

Valoración cualitativa:

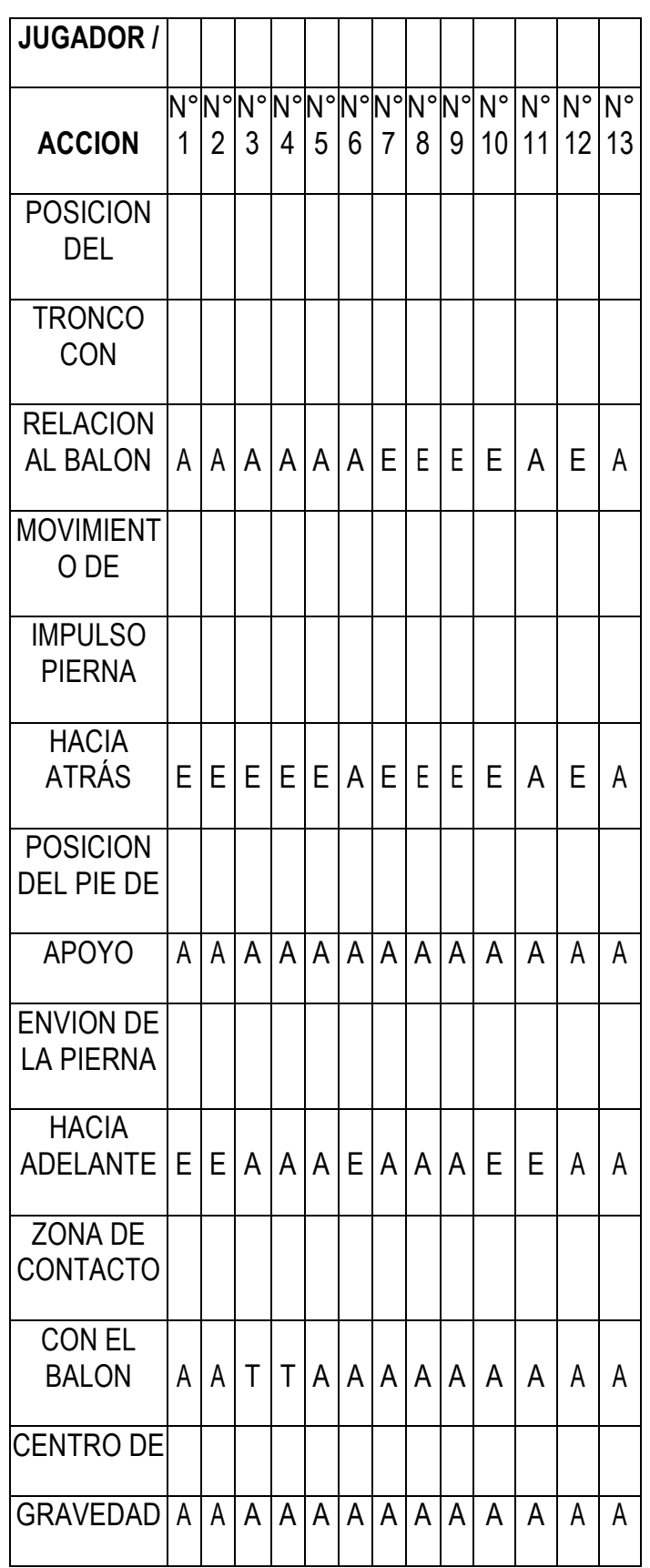

Valoracion cualitativa del Golpeo del balón con la parte interna del pie derecho

Fuente: Zambrano T. 2016

Analizando las gráficas de valoración, se puede evidenciar que los jugadores tienen un bajo nivel de eficacia a la hora de golpear el balón con la parte interna del pie derecho, solo 
4 de los 13 niños pudieron obtener entre el 30 y el $35 \%$ de eficiencia en la técnica, los otros 9 niños obtuvieron entre el 0 y el $20 \%$ de eficacia en la técnica. Cabe resaltar que la gran mayoría del grupo presenta dificultades en la postura del cuerpo con relación al balón, a veces se coloca el tronco muy adelante y a veces muy hacia atrás; también presentan inconvenientes cuando colocan el pie de apoyo, se observa que este no lo colocan en la distancia correcta con relación al balón, paralelamente a esto, se evidencio que la zona con que ellos impactan el balón en muchas ocasiones no es la más adecuada y por ende el balón no sale con la dirección y potencia deseada; en este contexto cabe subrayar que los 13 jugadores presentan problemas para manejar el centro de gravedad, lo que conlleva a no tener un balance adecuado del cuerpo.

Fuente: Zambrano T. 2016

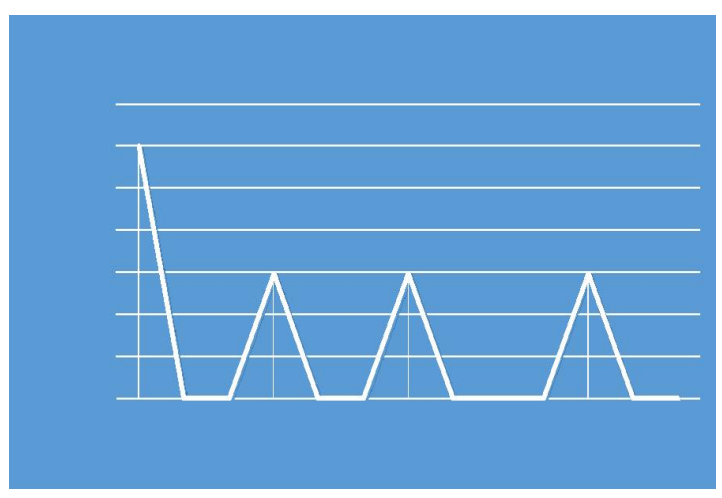

Fuente: Zambrano T. 2016

\section{VALORACION CUALITATIVA DEL GOLPEO DEL BALON CON LA PARTE INTERNA DEL PIE IZQUIERDO}



\begin{tabular}{|c|c|c|c|c|c|c|c|c|c|c|c|}
\hline BALON & \begin{tabular}{|l|l}
$\mathrm{A}$ &
\end{tabular} & & & A & & & & $\mathrm{A}$ & & & $A$ \\
\hline $\begin{array}{c}\text { MOVIMIENT } \\
\text { O DE }\end{array}$ & & & & & & & & & & & \\
\hline $\begin{array}{c}\text { IMPULSO } \\
\text { PIERNA }\end{array}$ & & & & & & & & & & & \\
\hline $\begin{array}{l}\text { HACIA } \\
\text { ATRÁS }\end{array}$ & $\mathrm{E}$ & & E & & A & $\mathrm{A} A$ & $A$ & A & $E$ & & A \\
\hline \begin{tabular}{|l|} 
POSICION \\
DEL PIE DE
\end{tabular} & & & & & & & & & & & \\
\hline APOYO & $\mathrm{A}$ & & A & A & $\mathrm{A}$ & $\bar{A} \bar{A}$ & A & $\mathrm{A}$ & $\bar{A}$ & & A \\
\hline $\begin{array}{l}\text { ENVION DE } \\
\text { LA PIERNA }\end{array}$ & & & & & & & & & & & \\
\hline $\begin{array}{c}\text { HACIA } \\
\text { ADELANTE }\end{array}$ & $\mathrm{E} \mid$ & & A & A & A & $\mathrm{A} \mid \mathrm{A}$ & $E$ & $E$ & $E$ & & A \\
\hline \begin{tabular}{|c|} 
ZONA DE \\
CONTACTO
\end{tabular} & & & & & & & & & & & \\
\hline $\begin{array}{l}\text { CON EL } \\
\text { BALON }\end{array}$ & A & A) & A & A & A & $\mathrm{A} A$ & A & A & A & & A \\
\hline $\begin{array}{c}\text { CENTRO } \\
\text { DE }\end{array}$ & & & & & & & & & & & \\
\hline VERAD & | & & A & $A$ & $A$ & & $A$ & $A$ & A & & A \\
\hline
\end{tabular}

\section{Fuente: Zambrano T. 2016}

en las gráficas y tablas de valoración del golpeo del balón con la parte interna del pie izquierdo se pudo comprobar que a excepción de un niño que obtuvo el $30 \%$ de eficacia en el test, los otros 12 niños obtuvieron entre el 0 y el $15 \%$, esto se debe a que como todos a excepción de uno manejan mejor la pateada con el pie derecho que con el izquierdo, se evidencian aún más dificultades que en las gráficas anteriores; como por ejemplo los 13 jugadores presentan seria dificultad para ubicar el tronco con relación al balón, además todos fallan en la colocación del pie de apoyo, ya que, unos lo sitúan muy cerca al balón y otros muy lejos del balón, asimismo, todos muestran falencias en la zona de contacto con el balón, esto se debe a que, el jugador no abre apropiadamente la cara interna del pie, por el contrario mantiene el pie más cerrado, en consecuencia el balón no sale con la dirección deseada, en relación con el centro de gravedad, se puede deducir que, los 13 jugadores presentan problemas a la hora de balancear el cuerpo, por ende, si la cadera no está en la posición adecuada, el gesto técnico no tendrá fluidez. Seguidamente se presentaran los datos correspondientes al golpeo del balón 
con la parte externa de las dos piernas:

\begin{tabular}{|c|c|c|c|c|c|c|c|c|c|c|c|}
\hline \multicolumn{12}{|c|}{ GOLPEO DEL BALON CON PARTE EXTERNA PIE DERECHO } \\
\hline \begin{tabular}{|l|}
$J U G$ \\
$A$
\end{tabular} & 1.5 & $\%$ & 1 & $\%$ & 1 & $\%$ & 0.5 & $\%$ & 0.5 & $\%$ & TOTAL \\
\hline $\mathrm{N}^{\circ} 1$ & A & 30 & X & 0 & $\mathrm{X}$ & 0 & $\mathrm{X}$ & 0 & $\mathrm{X}$ & 0 & 30 \\
\hline$N^{\circ} 2$ & $X$ & 0 & $A$ & 20 & $\mathrm{X}$ & 0 & $X$ & 0 & $\mathrm{X}$ & 0 & 20 \\
\hline $\mathrm{N}^{\circ} 3$ & $X$ & 0 & X & 0 & $A$ & 20 & $X$ & 0 & $X$ & 0 & 20 \\
\hline $\mathrm{N}^{\circ} 4$ & $x$ & 0 & $x$ & 0 & $x$ & 0 & $A$ & 15 & $\bar{X}$ & 0 & 15 \\
\hline $\mathrm{N}^{\circ} 5$ & $X$ & 0 & $x$ & 0 & $X$ & 0 & $A$ & 15 & $X$ & 0 & 15 \\
\hline $\mathrm{N}^{\circ} 6$ & $X$ & 0 & X & 0 & $A$ & 20 & $X$ & 0 & $X$ & 0 & 20 \\
\hline \begin{tabular}{|l|}
$\mathrm{N}^{\circ}$ \\
\end{tabular} & $A$ & 0 & $x$ & 0 & $X$ & 0 & $X$ & 0 & $A$ & 15 & 15 \\
\hline $\mathrm{N}^{\circ} 8$ & $X$ & 0 & $x$ & 0 & $A$ & 20 & $X$ & 0 & $X$ & 0 & 20 \\
\hline$\overline{N^{\circ} 9}$ & $X$ & 0 & X & 0 & $A$ & 20 & $X$ & 0 & $X$ & 0 & 20 \\
\hline $\begin{array}{l}N^{\circ} 1 \\
0\end{array}$ & $X$ & 0 & $A$ & 20 & $X$ & 0 & $X$ & 0 & $X$ & 0 & 20 \\
\hline $\begin{array}{l}N^{\circ} 1 \\
1\end{array}$ & $X$ & 0 & $X$ & 0 & A & 20 & $X$ & 0 & $X$ & 0 & 20 \\
\hline $\begin{array}{l}N^{\circ} 1 \\
2\end{array}$ & $X$ & 0 & 0 & 0 & $X$ & 0 & $X$ & 0 & A & 15 & 15 \\
\hline $\begin{array}{l}N^{\circ} 1 \\
3\end{array}$ & $x$ & 0 & $x$ & 0 & $x$ & 0 & $X$ & 0 & $A$ & 15 & 15 \\
\hline
\end{tabular}

Fuente: Zambrano T. 2016



Fuente: Zambrano T. 2016

En este diagnóstico se pudo observar a excepción de jugador que obtuvo el $30 \%$ de efectividad, el resto obtuvo entre el 0 y el $20 \%$, esto tiene su origen en una serie de dificultades que presenta el grupo en su gran mayoría, entre estas están la posición del pie de apoyo con respecto al balón, esta tiene una mayor dificultad que en el borde interno, debido a que como el pie con que se impacta el balón pasa muy cerca del otro que esta fijo en el suelo, al niño le da un poco de temor que en vez de patear el balón, se haga contacto con el pe de apoyo o con el suelo; es interesante examinar el problema desde la zona de contacto con el balón, ya que como el niño muy pocas veces golpean el balón con la parte externa, la gran mayoría termina por golpear el balón con la punta del pie, esto se debe a que el jugador no gira en su totalidad el tobillo, llevando como consecuencia a golpear el balón con la punta del pie o con el empeine; en lo que respecta al centro de gravedad, cabe decir que todos no realizan un balance adecuado del cuerpo ni inclinan la pelvis hacia la posición adecuada

\section{Resultados del pos test}

A continuación se presentaran los datos del postest para el golpeo del balón con la parte interna de las dos piernas:

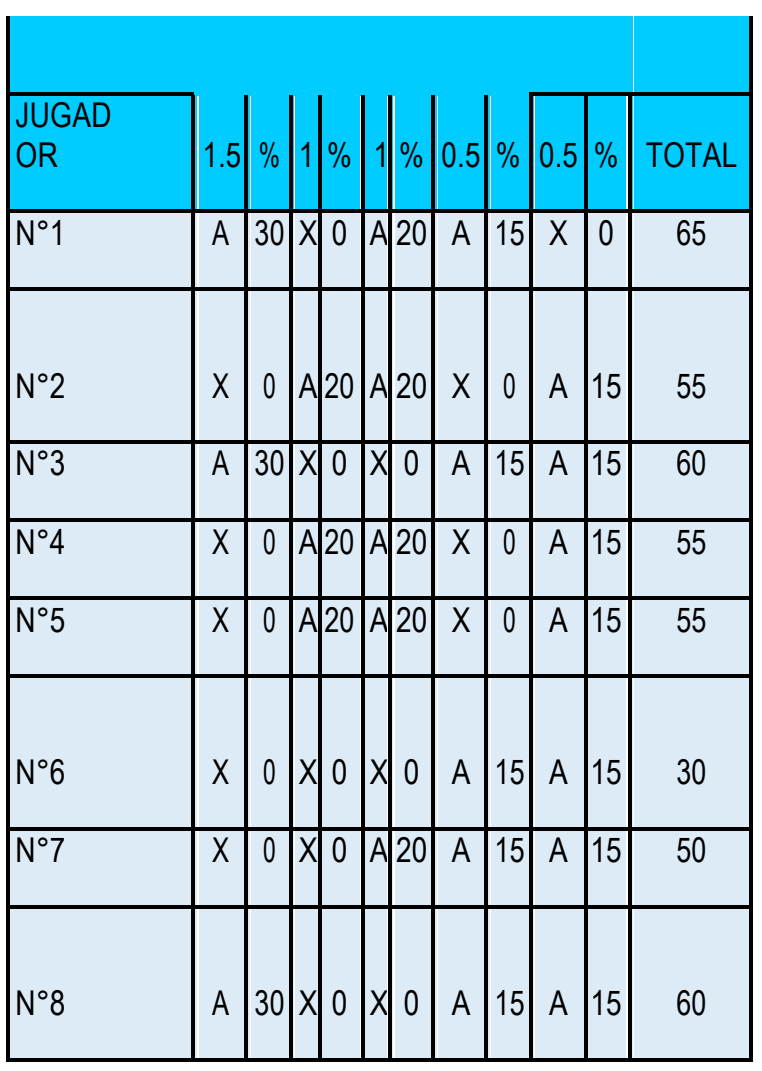




\begin{tabular}{|c|c|c|c|c|c|c|c|c|c|}
\hline $\mathrm{N}^{\circ} 9$ & A & $|30|$ & $A \mid 20$ & $|A| 0$ & $x$ & 15 & $x$ & $0 \mid$ & 65 \\
\hline$N^{\circ} 10$ & A & $|30|$ & $A \mid 20$ & \begin{tabular}{|l|l|}
$x$ & 0
\end{tabular} & $X$ & 0 & $X$ & 0 & 50 \\
\hline $\mathrm{N}^{\circ} 11$ & $x$ & 0 & A 20 & $A \mid 20$ & $\bar{X}$ & 0 & $\bar{X}$ & 0 & 40 \\
\hline$\overline{N^{\circ} 12}$ & $\mathrm{~A}$ & 30 & \begin{tabular}{l|l}
$x$ & 0
\end{tabular} & \begin{tabular}{|l|l|l|}
$x$ & 0
\end{tabular} & $x$ & 0 & $A$ & 15 & 45 \\
\hline$\overline{N^{\circ} 13}$ & A & 30 & 0 & A 20 & $x$ & 0 & $\bar{X}$ & 0 & 50 \\
\hline
\end{tabular}

Fuente: zambrano T. 2016

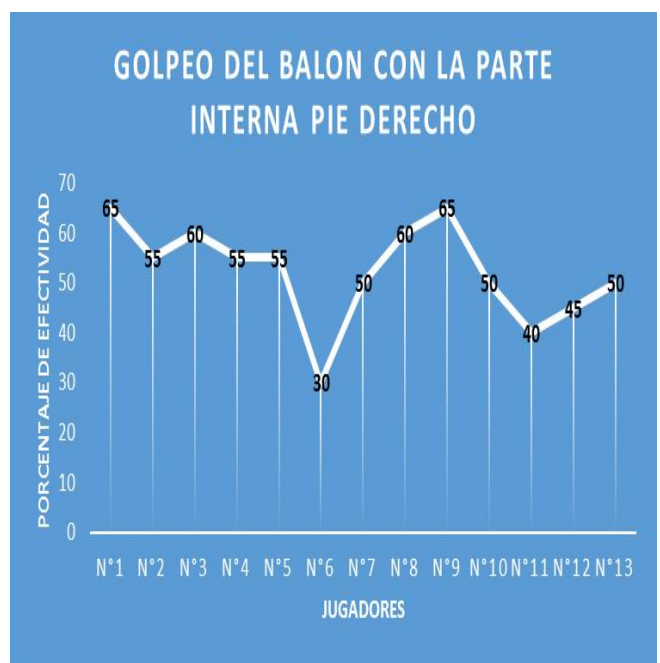

Fuente: Zambrano T. 2016

Analizando la valoración el golpeo con la parte interna del pie derecho, lo que se puede evidenciar en las dos primeras graficas es que el $77 \%$ de los jugadores obtuvieron entre el 50 y el $65 \%$ de efectividad y solo el $23 \%$ obtuvieron un puntaje de efectividad entre el 30 y el $40 \%$.

En lo que respecta, a lo cualitativo se logra evidenciar un buen nivel en cuanto a la ejecución de las posturas que componen los gestos técnicos, eso se puede deducir debido a que el $38,1 \%$ obtiene hasta 4 calificaciones de confección (C) lo que quiere decir que ejecutan el gesto técnico con una alta calidad. El 30,7\% obtuvo 2 y 3 calificativos de confección (C) es decir que ejecutan el gesto técnico con fluidez. $Y$ un restante $30,7 \%$ obtiene 1 calificativo de confección (C) y 5 calificativos de evolución (E) es decir que ejecuta el gesto técnico medianamente bien.

\section{GOLPEO DEL BALON CON PARTE INTERNA PIE IZQUIERDO}

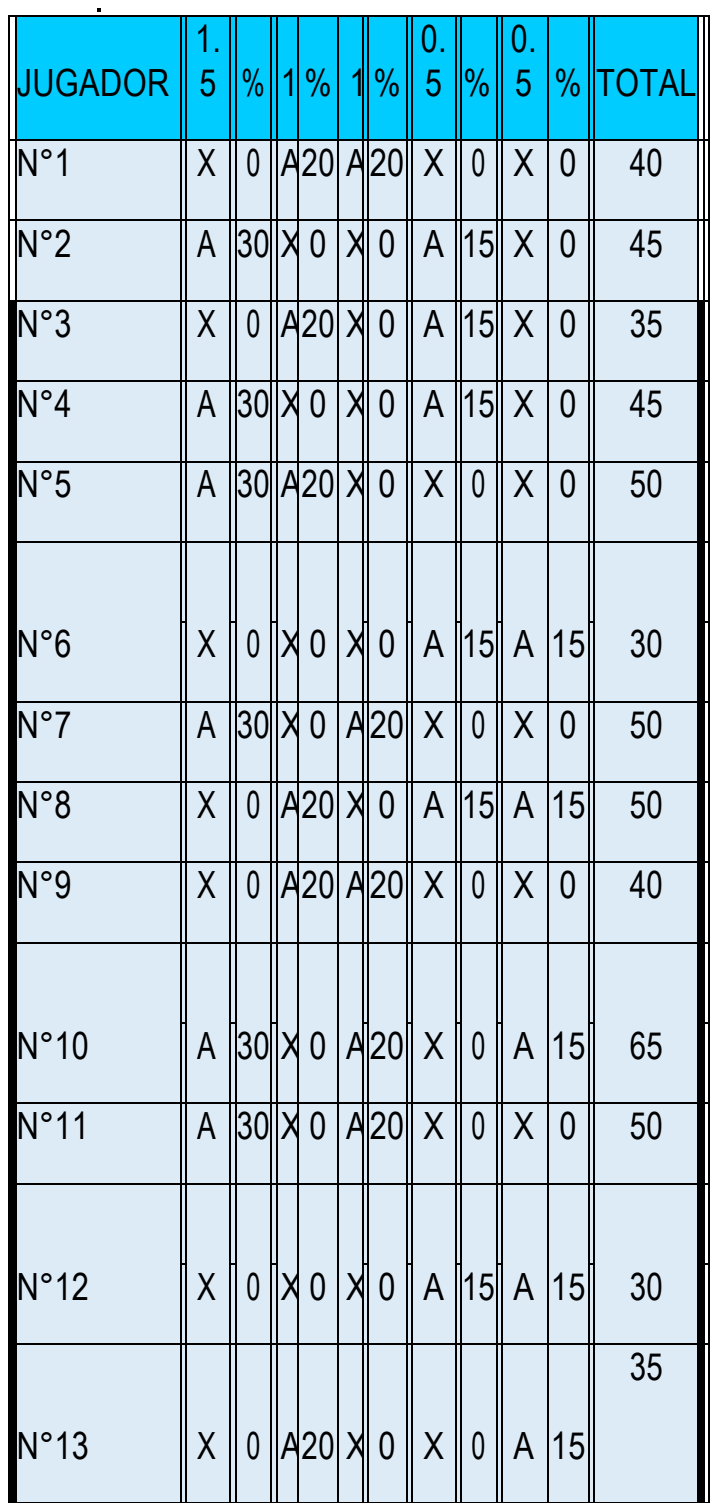

Fuente: Zambrano T. 2016

Observando la valoración cuantitativa del golpeo del balón con la parte interna del pie izquierdo, se puede apreciar que el $61,5 \%$ de los jugadores obtuvieron un nivel de efectividad que oscila entre el 40 y el $50 \%$; por otro lado el $30,7 \%$ obtuvo un nivel de efectividad que oscila entre el 30 y el $35 \%$ y solo un jugador obtuvo el $65 \%$ del nivel de efectividad.

En cuanto a la valoración cualitativa, se puede percibir que un solo jugador logra obtener 3 calificativos de confección (C) es decir que, ejecuta el gesto técnico de manera fluida. Por otra parte el $53,8 \%$ de los jugadores obtuvieron calificativos de evolución (E) es decir que ejecuta el gesto técnico medianamente bien. $Y$ el $38,4 \%$ de los jugadores obtuvieron calificativos de adyacencia (A) y evolución 
(E) es decir que ejecutan el gesto técnico medianamente bien aunque se evidencian dificultades en la posición del pie de apoyo, la zona de contacto con el balón no es la adecuada y el centro de gravedad es más alto de lo normal.

\section{DISCUSION}

De acuerdo con el con el desarrollo de la investigación y su evolución en cada una de sus etapas en la que se divide la estructura, se recopilaron afirmaciones de 13 autores que aportaron conceptos valiosos tanto en el modelo tradicional como en el modelo vertical y a su vez cuatro artículos investigativos, en los cuales se fundamenta la discusión de nuestro trabajo de investigación. Para lo previamente mencionado se tuvo en cuenta, la metodología empleada, los instrumentos para la medición, los autores que trataron sobre los diferentes modelos de aprendizaje, entre otros elementos que amparan las herramientas y el engranaje del trabajo de investigación aplicado a los niños de 12 años pertenecientes a la institución educativa Colegio Integrado Petrolea.

Destacando lo que dice thorpe (1992) citado por Abad (2005) sobre el modelo tradicional de aprendizaje, el cual prioriza una enseñanza analítica y separada la verdadera realidad del juego. Y lo que menciona Viciana y Delgado, (1999) afirmando que el profesor como metodología de trabajo se enfatiza de la repetición de un modelo a repetición del modelo aportado por él. A su vez utiliza estilos de enseñanza como mando directo, mando directo modificado y también la asignación de tareas a cada uno de los jugadores. Por otra parte Sáenz-López (1997) citados por Abad (2005) afirma que en la fase del calentamiento, este se realiza con los niños de forma específica.

Nuestro trabajo reserva una ajustada concordancia con los autores mencionados ya que, en la primera parte del macrociclo de trabajo se evidencia claramente como las sesiones de trabajo están dirigidas bajo un enfoque de pedagogía tradicional, es decir que el profesor explica mediante la utilización de medios como videos, fotografías, etc., como se ejecuta un gesto técnico para que luego el jugador trate de imitarlo y posteriormente ejecutarlo de manera correcta. Cabe destacar, que después de la primera fase ya mencionada del macrociclo de trabajo, viene una fase en la cual se le proporcionan en cada sesión de trabajo una serie de ejercicios los cuales incluido el calentamiento, son con elementos diferentes a un balón de futbol y están enfocados a ejercicios analíticos, es decir que los jugadores deben realizar los movimientos de acuerdo con la explicación precisa del profesor y a su vez tratar de imitar lo que este ejecuto en la demostración a través de el mismo o de alguna ayuda tecnológica.

De la misma manera, teniendo en cuenta lo que afirma López y Castejón, (1998 b) y Sampedro, (1999 a) citados por Abad (2005) en sus aportes al modelo vertical de aprendizaje en el futbol, estamos de acuerdo en que la técnica parece estar subordinada a la táctica y que no es suficiente solo con saber cómo hacer, si no que también el jugador debe saber cuándo y en qué momento hacer, de manera que pueda a través de un amplio bagaje técnico resolver las diferentes situaciones que se le presentan a lo largo de un juego.

Teniendo en cuenta las afirmaciones Giménez (2001); López y Castejón, (1998 a)y Wein, (1995 y 1998) citados por Abad (2005) las cuales apuntan a que se debe adaptar el futbol al niño y no al contrario. Tenemos afinidad con este pensamiento ya que si queremos que no hayan posibles deserciones en el futbol por parte de los niños a temprana edad, debemos dirigir las sesiones de trabajo hacia un enfoque vertical, es decir que el jugador trabaje la ejecución de los gestos técnicos con el objetivo no solo de automatizar un movimiento si no, con el propósito de tomar decisiones y adaptarse a las situaciones reales de un partido, $Y$ de hecho a partir del segundo mesociclo se introdujo las acciones combinadas y se le dio vital importancia al juego para que el niño se desenvuelva en un ambiente agradable de aprendizaje. Lo que conlleva a que este pueda no solo a ejecutar un gesto técnico de forma esquematizada, si no que también sepa qué hacer con todo ese bagaje en las acciones combinadas.

En contraste con lo dicho por los autores anteriores, los cuales hicieron valiosos aportes tanto a la pedagogía analítica, como a la vertical, me gustaría dejar claro que, discrepo en el sentido de que se tenga que utilizar un solo método de enseñanza sea vertical 0 tradicional. Por el contrario, estamos de acuerdo con las afirmaciones de Ávila y Chirosa (1997) y 
Sans, Frattarola y Sagrera (1999) citados por Abad (2005) las cuales apuntan a que no se excluyen de forma absoluta el modelo tradicional de enseñanza ni los ejercicios analíticos, sino que se advierte de que por sí solos no son suficientes, por lo tanto en nuestra investigación implementamos una fusión entre el modelo tradicional y el modelo vertical/global. Lo cual le permite al jugador mediante la el primer modelo adquirir una automatización de los movimientos que componen el gesto técnico, para que luego en el segundo modelo aprenda que hacer y en qué momento puede darle el uso adecuado a cada golpeo con la parte del pie que corresponda en el momento.

Los investigadores Jimenez, Marin, Ramon, Aguilar; Grajales y Ramirez (2010) En su artículo: Efectos de un plan de aprendizaje estructurado mediante la práctica por bloques en el golpeo con la parte interna del pie en niños de 10 a 12 años de edad diseñaron un plan de entrenamiento por bloques enfocado hacia el golpeo del balón con la parte interna del pie, realizando 1000 golpeos del balón mediante este gesto técnico presentaron diferencias significativas entre el pretest y el postest obteniendo asi una mejoría en la ejecución del golpeo con la parte interna del pie Paralelamente a lo planteado por estos autores, nosotros implementamos una alternativa metodológica basada en la fusión de dos modelos de aprendizaje en la cual, no solo se trabajó el gesto técnico del golpeo del balón con la parte interna del pie, sino que, también se introdujo el gesto técnico con el empeine y la parte externa del pie, además se enfatizó en el trabajo con las dos piernas; la cual, luego del análisis estadístico se puede apreciar que la ejecución tanto del golpeo con la parte interna y externa arrojaron un $p$ valor de 0,0000 presentando así diferencias significativas entre el pretest y el postest. Obteniendo así al igual que la anterior investigación una mejoría con nuestra propuesta metodológica.

Ahora bien, observando el trabajo de investigación de Vera y Mariño (2008), los cuales diseñaron una preparación técnico táctica dirigida a niños de 13 y 14 años, la cual incluyeron una tabla en la que se puede valorar cualitativamente los diferentes movimientos que realiza un futbolista.

Lo anterior evidencia una correlación con nuestro trabajo debido a que diseñamos una tabla de valoración cualitativa la cual permite analizar y calificar la ejecución de las diferentes acciones que componen un gesto técnico.

Ahora bien, en el trabajo de Merchan (2010), se puede apreciar que se diseña e implementa una propuesta elaborada en base a la metodología global teniendo como instrumento de medición el de tiro a portería (Roberto Montes-bateria test), esta investigación como se mencionó anteriormente se realizó de manera que el enfoque fuese más hacia la pedagogía global y no tanto a la analítica. Luego de haber realizado el pre y el postest evidenciaron diferencias significativas luego de haber aplicado la propuesta metodológica, lo cual, obtuvo como resultado una mejoría en la eficacia de la técnica del jugador.

Lo anterior, permite apreciar una afinidad con nuestro trabajo de investigación dado que, aquí también se midió sobretodo el tiro con el empeine de las dos piernas, a través del test de Roberto Montes. Lo cual después de haberle aplicado la estrategia metodológica, se puede evidenciar a través del análisis estadístico que se presentaron diferencias significativas entre el pretest y el postest en la valoración cuantitativa del golpeo del balón con el empeine de las dos piernas. Lo cual quiere decir que, no solo una estrategia metodológica en base a la pedagogía global sirve para mejorar el nivel de eficacia de la técnica; si no que también como alternativa, se puede tomar como referencia el modelo tradicional y el modelo vertical que a su vez comprende la pedagogía global y fusionarlos para que también se pueda obtener una mejoría en la técnica en el futbol.

Teniendo en cuenta la investigación de Correa, Robledo y Jimenez, (2009) la cual se basó en un plan de entrenamiento para trabajar el golpeo del balón con el empeine, basado en la pedagogía global y que fue aplicada en niños de 12 y 13 años en la ciudad de Medellín. Lo cual tuvo como resultado en la comparación entre el pretest y el postest, que no existen diferencias significativas, lo cual se entiende que la planificación en base a la metodología global no tuvo incidencia en el aprendizaje del gesto técnico del golpeo del balón con el empeine.

Lo previamente, expuesto discrepa con nuestra investigación en el sentido que, podemos decir que la propuesta metodológica aplicada, en lo que respecta al golpeo del balón con el 
empeine, la cual fue una de las tres superficies de contacto que se tuvieron en cuenta para realizar nuestra propuesta metodológica, que aunque evidencia poca mejoría en la ejecución del gesto técnico; si se puede apreciar diferencias significativas en el nivel de efectividad. Es decir que se obtuvo una mejoría considerable en el nivel de efectividad del golpeo del balón con el empeine.

\section{CONCLUSIONES}

Realizando un barrido de las diferentes bases de datos bibliográficas nacionales e internacionales y a través de revistas indexadas como: Apunts: educación físical esports ,Educación física y deporte/udea, Revista de psicología del deporte, Dialnet/unirioja, Sports science, entre otras, trabajos de maestría e incluso de doctorado, se obtuvo como logro la realización de un análisis teórico, el cual muestra como se ha venido trabajando a través de los años la enseñanza de la técnica en el futbol en las categorías menores.

Si bien se hallaron similitudes en algunos trabajos investigativos, los cuales se enfocaron en trabajar el golpeo del balón con el empeine, con la parte interna, trabajos con pierna no dominante y pierna dominante, entre otros componentes, no se encontraron registros en los cuales su planificación sea a través de una fusión de metodología tradicional y metodología vertical/global, además, tampoco se halló, dentro de estas investigaciones una evaluación cualitativa y cuantitativa del golpeo del balón con la parte interna, externa y empeine de las dos piernas, así mismo que en el macrociclo se introdujeran trabajos con materiales diferentes a un balón de futbol. Igualmente tampoco se presenciaron trabas en los cuales se le diera vital importancia al golpeo del balón con la parte externa de las dos piernas.

La aplicación de dos test específicos como lo son el test de tiro a portería (Montes, 2006) y el test técnico para futbol de la Universidad del Valle, y las tablas de valoración cualitativa para apreciar el nivel de ejecución del gesto técnico del golpeo del balón con la parte interna, externa y el empeine de las dos piernas, las cuales fueron validadas por dos expertos en futbol de categorías menores, proporcionaron con autenticidad y confiabilidad, el nivel tanto de efectividad como el nivel de ejecución de los gestos técnicos para golpear el balón con el pie.

Se elaboró una estrategia metodológica basada en la fusión de dos grandes modelos de aprendizaje los cuales son el tradicional 0 analítico y el modelo vertical/global dentro de un diseño cuasi-experimental, a su vez teniendo presente las fases de diagnóstico, diseño de la estructura de la estrategia metodológica, y la fase de intervención la cual después de haberse aplicado durante cinco meses y trabajando tres veces por semana se pudo evidenciar que luego de haberse realizado el análisis estadístico, en la parte cuantitativa se obtuvo como resultado un $p$-valor del 0,000, lo cual, quiere decir que, después de haber aplicado la estrategia metodológica, hubo una evolución en cuanto al nivel de efectividad del golpeo con las partes del pie trabajadas.

En la parte cualitativa, se puede evidenciar después del análisis estadístico, mejoría considerable en las acciones que componen el gesto técnico para el golpeo del balón con la parte interna y externa de las dos piernas, y el empeine, sin embargo cabe resaltar que a pesar de que en el postest se obtuvo un p-valor del 0,9532 para el pie izquierdo, es decir que en la prueba global no se obtuvieron diferencias significativas, sin embargo si observamos acción por acción si se evidencian diferencias significativas; ahora bien, mirando el golpeo con el empeine del pie derecho obtuvo un p-valor del 0.008 , lo cual evidencia diferencias significativas entre el pretest y el postest, y también si miramos acción por acción, también se evidencian notables mejorías en cada una de estas.

\section{REFERENCIAS BIBLIOGRÁFICAS}

BENEDEK, E. (2001). FUTBOL INFANTIL. BARCELONA: PAIDOTRIBO.

BENITEZ, R. (1982). Cuándo empezar la iniciación al fútbol.

http://www.rafabenitez.com/web/index.php? act=mostrarContenidos\&idioma $=e s \& c a=17$.

Blasco, V. J. (2007). Construcción de una relación entre algunos conceptos epistemológicos de la Educación Física (Praxiología Motriz). EF DEPORTES.

BRÜGGEMANN, D. (2004). FUTBOL. ENTRENAMIENTO PARA NIÑOS Y JOVENES. BARCELONA: PAIDOTRIBO. 
Clara Isabel Pazo Haro, P. S.-L. (2011). La formación de los jugadores de fútbol de alta competición desde la perspectiva de los coordinadores de cantera. WWW.REVISTA-APUNTS.COM.

CORTEZ, C. E. (1981). METODOLOGIA PROPIA DE LA ENSEÑANZA DEL FUTBOL. UDEA.EDU.CO/REVISTA/REVISTA EDUCACION FISICA Y DEPORTE.

DANIEL CARMONA CUEVAS, J. F. (2015). EFECTOS DE UN PROGRAMA DE FORMULACIÓN DE OBJETIVOS $Y$ MOLDEAMIENTO DEL PASE EN JÓVENES JUGADORES DE FÚTBOL. REVISTA DE PSICOLOGIA DEL DEPORTE.

Daniela Tursi, S. N. (2013). Video analysis as an instrument in juvenile soccer training. DIALNET/ UNIRIOJA.

Daniela Tursi, S. N. (2013). Video analysis as an instrument in juvenile soccer training. journal of human sport and excercise.

Echevarría, D. L. (2006). El fútbol 9 como alternativa al fútbol 11, a partir del estudio de la utilización del espacio de juego. WWW.REVISTA-APUNTS.COM.

Elena Escolano-Pérez, M. L.-N.-E. (2014). La resolución de problemas como estrategia de enseñanza para el aprendizaje de la táctica ofensiva en la categoría prebenjamín de fútbol 8. REVISTA PSICOLOGICA DEL DEPORTE.

Elkin Alberto Arias Arias, J. O. (2004). Comparación entre la práctica aleatoria y la práctica por bloques en dos fundamentos técnicos del fútbol en niños de 6 a 8 años. DIALNET/ UNIRIOJA.

Gastón González Sepúlveda, C. O. (2010). ENTRENAMIENTO ESPECÍFICO DEL BALANCE POSTURAL EN JUGADORES JUVENILES DE FÚTBOL. REVISA INTERNACIONAL DE MEDICINA Y CIENCIAS DE LA ACTIVIDAD FISICA Y EL DEPORTET.

Jiménez, D. L. (2010). Adaptando la competición en la iniciación al fútbol: estudio comparativo de las modalidades de fútbol 3 y fútbol 5 en categoría prebenjamín. WWW.REVISTAAPUNTS.COM.

José Carlos Fernández García, A. R. (2006). Análisis de la iniciación al fútbol. DIALNET/ UNIRIOJA.

Juan Osvaldo Jiménez Trujillo, F. H. (s.f.). Efectos de un plan de aprendizaje estructurado mediante la práctica por bloques en el golpeo con la parte interna del pie en niños de 10 a 12 años de edad. dialnet/ unirioja.
Kassim, M. (2014). Towards the reflective football coach from qualitative analysis. journal of human sport and excercise.

Lozano, D. O. (2005). La Ciencia de la Acción Motriz, un paradigma en continua evolución. EF DEPORTES.

Paulomi Das, P. C. (2013). Urban-rural contrasts in motor fitness components of youngster footballers in West Bengal, India. dialnet/ unirioja.

PAZZIN, T. R. (2014). Escolas de futebol: uma pesquisa sobre a formação dos treinadores e metodologia utilizada nas escolas de futebol de cidades do litoral norte do Rio Grande do Sul. Revista Brasileira de Futsal e Futebol.

PEITERSEN, B. (2003). TECNICA DEL FUTBOL: EL ABC DEL ENTRENAMIENTO JUVENIL. BARCELONA: PAIDOTRIBO.

Pérez, R. C. (2014). Incidencia de diferentes metodologías de enseñanza-entrenamiento en el fútbol base sobre indicadores del abandono deportivo. dialnet/ unirioja.

RAFAEL ENRIQUE LOZANO ZAPATA, Y. B. (2013). DETERMINACIÓN Y VALORACIÓN CINEMÁTICA EN EL LANZAMIENTO DEL TIRO LIBRE PREFERENCIAL EN EL FUTBOL. REVISTA ACTIVIDAD FISICA Y DESARROLLO HUMANO.

RIERA, J. R. (2005). FUNDAMENTOS DEL APRENDIZAJE DE LA TECNICA Y LA TACTICA DEPORTIVA. BARCELONA: INDE PUBLICACONES.

Robles, M. T. (2005). MODELOS DE ENSEÑANZA DE FÚTBOL. www.wanceulen.com/revistaef-digital/nos.../articulo\%201-9.htm.

ROBLES, M. T. (2011). MODELOS DE ENSEÑANA DEL FUTBOL. es.scribd.com/doc/70883447/MODELOSDE-ENSENANZA-DE-FUTBOL.

Rodríguez, F. J.-G. (2010). El proceso de formación del jugador durante la etapa de iniciación deportiva. www.revista-apunts.com.

rodriguez, j. (2010). La iniciación deportiva en el contexto escolar: análisis de los modelos de enseñanza. ef deportes, 1.

Sáenz, S. S. (2009). Validación de un protocolo para la medición de la velocidad de golpeo en fútbol. www.revista-apunts.com.

Safaríková, S. (2012). The influence of football on the personal development of children in Uganda. DIALNET/ UNIRIOJA.

Salinero, J., González-Millán, C., Ruiz-Vicente, D., Abián Vicén, J. G.-A., \& RodríguezCabrero, M. y. (2011). Valoración de la condición física y técnica en fútbolistas jóvenes. revista internacional de medicina $y$ ciencias de la actividad fisica y el deporte.

Sixto González Víllora, L. M. (2011). Conocimiento táctico y la toma de decisiones en jóvenes 
jugadores de fútbol (10 años). REVISTA PSICOLOGICA DEL DEPORTE.

WEIN, H. (2004). futbol a la medida del niño. barcelona: ino publicaciones.

WEINECK, J. (2005). ENTRENAMIENTO TOTAL. BARCELONA: PAIDOTRIBO.

Zhelyazkov, T. (2001). bases del entrenamiento deportivo. Barcelona: paidotribo.

Zubiaur, M. (2005). Algunas consideraciones sobre la utilización del modelado en la adquisición de habilidades motrices en ninos. REVISTA 
